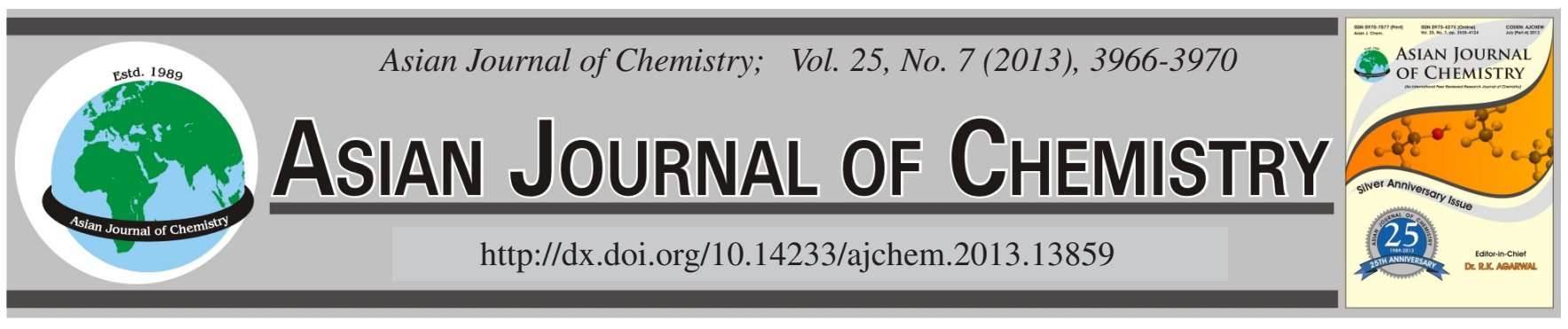

\title{
Inverse Emulsion Polymerization of Dimethyl Diallyl Ammonium Chloride and Acrylamide for Water Treatment
}

\author{
Peng Zhang* and Bozhi Ren
}

College of Civil Engineering, Hunan University of Science and Technology, Xiangtan 411201, Hunan Province, P.R. China

*Corresponding author: Tel./Fax: +86 731 58290052; E-mail: zhangpeng388@126.com

\begin{abstract}
Polymerization of dimethyl diallyl ammonium chloride and acrylamide (PDA) has been formed by using industrial dimethyl diallyl ammonium chloride (DMDAAC) and industrial acrylamide (AM) initiated by $\mathrm{K}_{2} \mathrm{~S}_{2} \mathrm{O}_{8}-\mathrm{NaHSO}_{3}$. The effect of reaction variables such as concentration of the reactants, reactants ratio, dosage of EDTA and reaction time were investigated and the product was detected and characterized with IR spectroscopy and thermo gravimetric analysis. The DBP removal efficiency by PDA was investigated. In the batch mode of studies, the $\mathrm{pH}$ value and effect of the intrinsic viscosity of product were investigated and when the initial concentration of simulated water was $0.5 \mathrm{mg} / \mathrm{L}, \mathrm{pH}$ value of 10 , dosage of PDA was $8 \mathrm{mg} / \mathrm{L}$, the removal efficiency of DBP reached a maximum value $92.4 \%$
\end{abstract}

Key Words: Water treatment, Phthalate acid esters (PAEs), Flocculant, Endocrine disrupting compound.

\section{INTRODUCTION}

Flocculant is one of the key factors in the flocculation process ${ }^{1}$. Organic polymer flocculant is a kind of water and wastewater treatment reagent, its efficiency is superior to the inorganic polymer flocculants and its remarkable characteristics is a higher rate of settlement, low dose, the formed floes are large and tenacious and it have been developed rapidly $^{2}$. Among them polymerization of diallyl dimethyl ammonium chloride and acrylamide (PDA) is one of the typical kinds and has become most widely used. The organic flocculant PDA was grafted by free radical polymerisation of acrylamide monomer (AM) onto the cationic monomer dimethyl diallyl ammonium chloride (DMDAAC), which widely used in the field of oil exploration, paper making, textile printing, industrial wastewater treatment and sludge dewatering, etc. ${ }^{3,4}$.

Nowadays, the appearance of endocrine disrupting compounds in the aquatic environment has become more and more noted, sparking discussion between scientists, politicians, industrial and environmental organizations on the significance of the effect on wildlife and humans ${ }^{5}$. Phthalate acid esters (PAEs) are a class of chemical compounds most widely used in industrial production belong to endocrine disrupting compounds. Their major applications are as plasticizers for polyvinyl chloride (PVC) resins, adhesives and cellulose coating (ca. $85 \%$ of the whole production $)^{9}$. The current worldwide yield of phthalate acid esters has reached 2.7 million metric tonnes per annum ${ }^{6,7}$. The large production and utilization caused a significant spread of these compounds into the environmental. Pollution of waters due to phthalate acid esters is a serious environmental and public health issue. Esters have been found to accumulate in the environment. In order to prevent environmental pollution and/or infection of drinking water sources the discharge criteria of wastewater are becoming stricter according to new legislations in force ${ }^{8,9}$. Thus, the removal of phthalate acid esters from water has become important to maintain water quality within the standard suitable for environmental and human health. Since phthalate acid esters are difficult to degrade biologically and photo-chemically, there is need to explore other treatment process for their removal.

Coagulation-flocculation process is an important environmental protection technology that finds a wide range of application in water or wastewater treatment facilities ${ }^{10-12}$. The flocculating effect is determined mainly by the qualities and species of flocculants ${ }^{13-16}$. Therefore, it is worth investigating the possibility of the same being successful in the removal of di- $n$-butyl phthalate from water.

In this study, polymerization of dimethyl diallyl ammonium chloride and acrylamide (PDA) was synthesized by the graft copolymerization of acrylamide (AM) over dimethyl diallyl ammonium chloride (DMDAAC). The structure of PDA was characterized by IR and DTA, TG techniques, respectively. The effects of reaction variables such as dosage, kind of initiator, total concentration of the reactants, reactants ratio, 
dosage of EDTA and the reaction time on polymerization were studied. Lastly, the DBP removal rate of the flocculating agent on simulated wastewater solutions were also studied.

\section{EXPERIMENTAL}

Analytical grade di- $n$-butyl phthalate was purchased from Chongqing Oriental reagent factory; HPLC grade methanol was purchased from Shandong yuwang industrial Co., Ltd. chemical branch; Industrial grade dimethyl diallyl ammonium chloride (DMDAAC) and acrylamide (AM) was purchased from Chongqing Blue Clean Water Material Co., Ltd.; De-ionized water was used throughout the experiment and $\mathrm{pH}$ value of solution was adjusted using $\mathrm{NaOH}$ and $\mathrm{HCl}$ solution.

Instruments used in the experimental setup were as follows: Fourier transformed infrared (FT-IR) spectrophotometer supplied by IR Prestiger-21 from Japan; TGA/DSC1/ 1100LF supplied by Mettler-Toledo Instruments Co., Ltd. from Switzerland; ZR4-6 Jar Tester supplied by Zhongrun Water Industry Technology Development Co. Ltd. from Shenzhen, China; high performance liquid chromatograph(LC-10AT) supplied by Shimadzu.

Synthesis procedure of PDA and purification of the product: A three-necked, round-bottom flask $(250 \mathrm{~mL})$ was used as the reaction vessel. A given mass of Span 80-Tween 80 and liquid paraffin were added to the flask and well-mixed. Then followed by adding the proportion of AM monomer aqueous solution and liquid DMDAAC monomer, high-speed stirring, then add a certain concentration of hydrogen sulfite aqueous solution of sodium $\left(\mathrm{NaHSO}_{3}\right)$, potassium persulfate solution $\left(\mathrm{K}_{2} \mathrm{~S}_{2} \mathrm{O}_{8}\right)$ and DETA solution, emulsion $20 \mathrm{~min}$ and introduced nitrogen into the reaction vessel $0.5 \mathrm{~h}$ and controlling the temperature at $40^{\circ} \mathrm{C}$ in a water bath. After the reaction time, the copolymer was washed with fresh acetone for several time to remove the unreacted monomers, at last washed with ethanol. The product was finally dried under vacuum at $65^{\circ} \mathrm{C}$ to constant weight.

Wastewater sample: Take $1 \mathrm{~mL} \mathrm{DBP}$ with the pipette, place it in a $1000 \mathrm{~mL}$ volumetric flask, dissolved in chromatographically grade methanol and then constant volume to the mark with distilled water and configured to $1000 \mathrm{mg} / \mathrm{L}$ a single standard stock solution, stored in a refrigerator at $4{ }^{\circ} \mathrm{C}$ and set aside. Then take $0.5 \mathrm{~mL}$ DBP single standard stock solution with the pipette 7 , placed in a $1 \mathrm{~L}$ volumetric flask, then constant volume to the mark with distilled water and configured to 0.5 $\mathrm{mg} / \mathrm{L}$ model water solution with the current, to avoid prolonged exposure in light conditions.

Adsorbing and removal of DMP with DPB: A ZR 4-6 stirring machine (Shenzhen Zhongran Water Industry Technology Development Co., Ltd., Shenzhen, China) with six stirrers was used in this experiment. $100 \mathrm{~mL}$ of wastewater was transferred into a beaker. Flocculants were dosed under medium stirring speed of $300 \mathrm{r} / \mathrm{min}$ for $1 \mathrm{~min}$ and then changed to the speed of $160 \mathrm{r} / \mathrm{min}$; after $4 \mathrm{~min}$, the speed was reduced to a slow stirring speed of $40 \mathrm{r} / \mathrm{min}$ for $5 \mathrm{~min}$. After, quiescent settling of $0.5 \mathrm{~h}$, samples were collected from $2 \mathrm{~cm}$ below the surface for measurement of DBP using an HPLC.

\section{RESULTS AND DISCUSSION}

Preparation conditions of PDA: Effect of the concentration of the initiator on the intrinsic viscosity when the other reaction variables were fixed, the effect of the concentration of the initiator on the intrinsic viscosity was studied.

Fig. 1 shows that the optimum concentration of the initiator $\left(\mathrm{NaHSO}_{3}-\mathrm{K}_{2} \mathrm{~S}_{2} \mathrm{O}_{8}\right)$ was $0.03 \%$. With the increased in the amount of initiator, the intrinsic viscosity of the product increased firstly and then decreased and the largest intrinsic viscosity was $6.21 \mathrm{dL} / \mathrm{g}$. The reason may be that when the initiator concentration was low, the initiator leaded to low efficiency as the solvent cage effect, also leaded the polymerization reaction was slow, so that the product of intrinsic viscosity value was low. When the initiator dosage increased, there was enough initiator to meet the induced decomposition and the cage effect decomposition, intrinsic viscosity of the product reached the maximum. But when the initiator excessived, resulting in the number of free radicals too much, the reaction system in the formation of the active center increased their mutual collision probability of the termination of the reaction increased and the chance of chain termination increased, shorten the molecular chain of the product while lead to the product of intrinsic viscosity decreased.

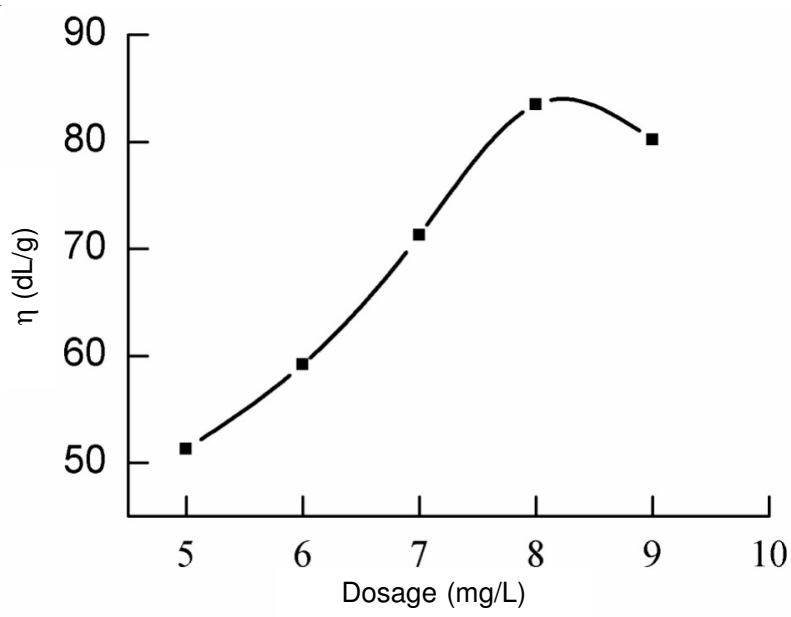

Fig. 1. Effect of the concentration of initiator on the intrinsic viscosity

Effect of the reactants ratio on the intrinsic viscosity: When the other reaction variables were fixed, the effect of the reactants ratio on the intrinsic viscosity was studied.

Fig. 2 shows that the optimum ratio of DMDAAC to AM was $2: 8$ and the intrinsic viscosity of the product was $7.41 \mathrm{dL} / \mathrm{g}$. That because the polymerization was free radical copolymerization reaction, when increasd the concentration of the DMDAAC, the free radicals could also increased, so the intrinsic viscosity of the product increased, but when free radicals achieved to a certain extent, the free radicals disappeared by the inter-collision, resulted in the reaction limited, so the best ratio of monomer DMDAAC and AM was 2:8.

Effect of the dosage of EDTA on the intrinsic viscosity: When the other reaction variables were fixed, the effect of the dosage of EDTA on the intrinsic viscosity was studied.

Fig. 3 shows that the intrinsic viscosity of polymers first increased and then decreased with the increased of the amount of EDTA. When the EDTA dosage was $0.60 \mathrm{~mL}(1.0 \mathrm{~mol} / \mathrm{L})$, the intrinsic viscosity of the product could reached maximum, which was $5.63 \mathrm{dL} / \mathrm{g}$. That was due to EDTA could be multivalent metal ions, which reduced adverse reaction. The 


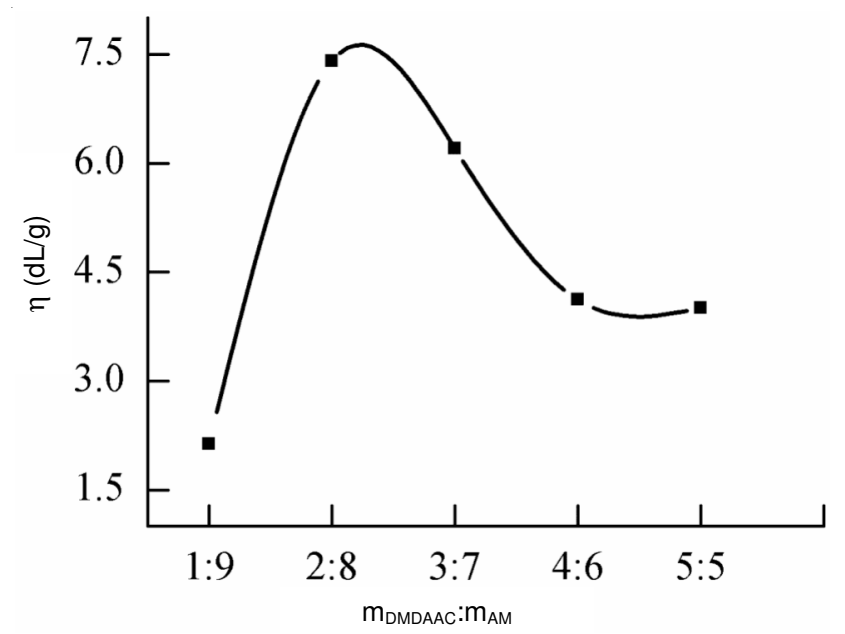

Fig. 2. Effect of the reactants ratio on the intrinsic viscosity

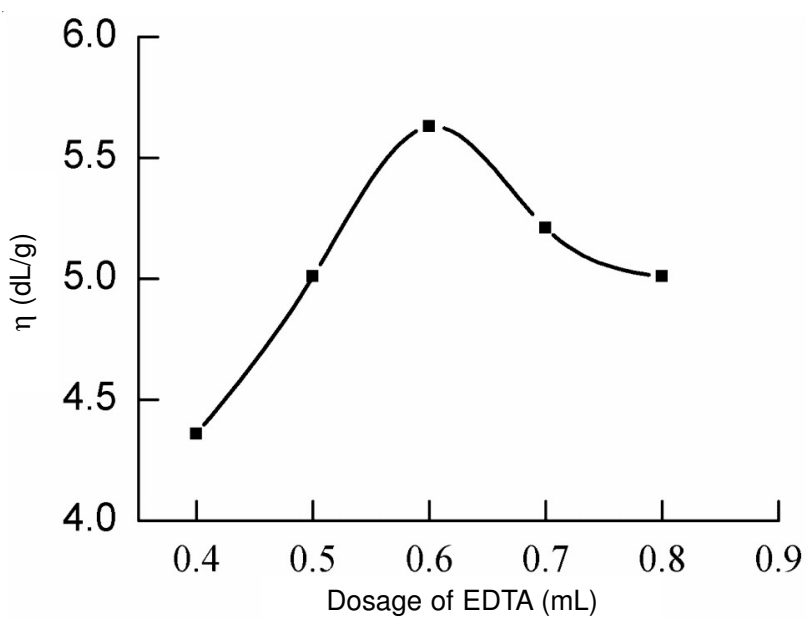

Fig. 3. Effect of dosage of DETA on the intrinsic viscosity

experiment used monomers were industrial products, containing certain metal ions, if not join the complexing agent EDTA, impurities in the monomer would participate in the polymerization reaction, so that the polymerization limited. Although EDTA was able to shield the interference of impurities inside the monomer polymerization, leaded the product of intrinsic viscosity increased, meanwhile EDTA also could make the growth of chain transfer, so if the EDTA excessed, the intrinsic viscosity of the product would be decreased.

Effect of the reaction time on the intrinsic viscosity: When the other reaction variables were fixed, the effect of the temperature on the intrinsic viscosity was studied.

Fig. 4 shows that with the increased of the reaction time, the intrinsic viscosity of the product increased firstly and then stable. That was because prolonging the reaction time resulted in the growth of monomer conversion rate. Continuing to extend the reaction time could not increase in intrinsic viscosity obviously. Therefore the optimum reaction time was $4 \mathrm{~h}$.

\section{Characterization of PDA}

IR spectra: IR spectra of PDA was analyzed using the Fourier transformed infrared (FT-IR) spectrophotometer supplied by IR Prestiger-21 from Japan, in the range of 4000$500 \mathrm{~cm}^{-1}$ with $\mathrm{KBr}$ as dispersant. The IR spectra macrograph is shown in Fig. 5.

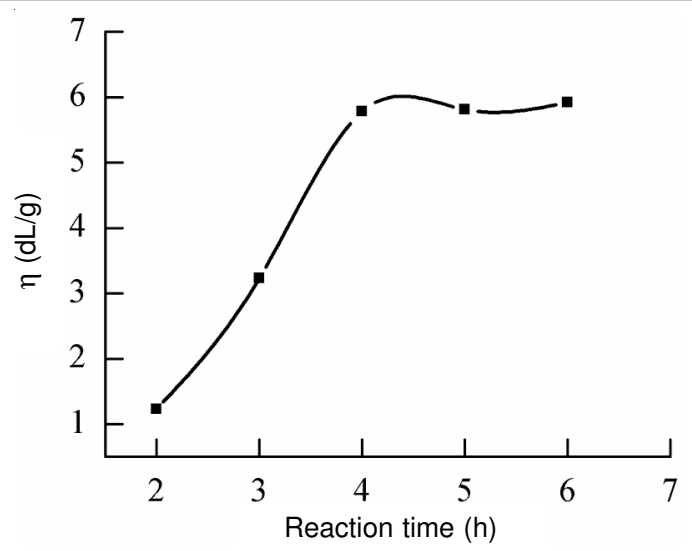

Fig. 4. Effect of the reaction time on the intrinsic viscosity

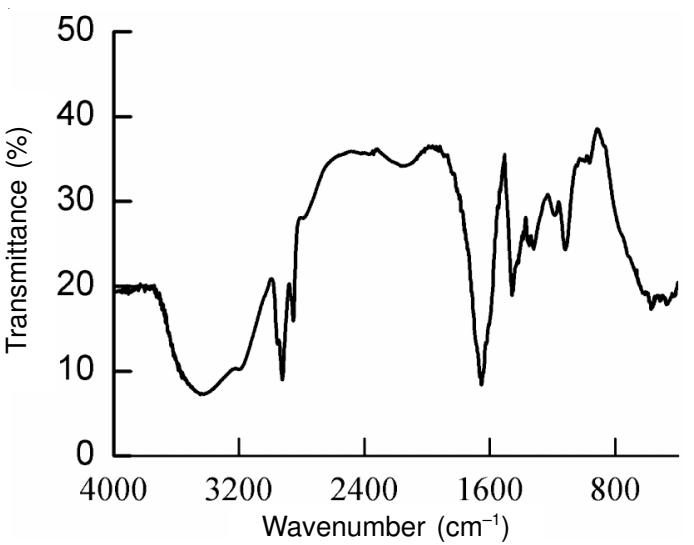

Fig. 5. Infrared spectroscopy chart of PDA

It was evident that the absorption peak at $3447 \mathrm{~cm}^{-1}$ correspond to $-\mathrm{NH}_{2}$ stretching vibration for acrylamide, the stretching vibration nearby $2924 \mathrm{~cm}^{-1}$ attributable to the $-\mathrm{CH}_{3}$ connected with $-\mathrm{N}^{+}$of DMDAAC, the characteristic peak at $2865 \mathrm{~cm}^{-1}$ was unsaturated- $\mathrm{CH}_{2}$ absorption peak and the characteristic peak at $1651 \mathrm{~cm}^{-1}$ was $-\mathrm{CONH}_{2}$ absorption peak, the characteristic peak at 1460 and $1651 \mathrm{~cm}^{-1}$ was the six stretching vibration of the DMDAAC polymerization which proved the polymer had six heterocyclic. FIRT characterization results showed that the synthesized product is a copolymer of AM and DMDAAC.

Thermogravimetric analysis: Fig. 6 shows the thermal stability of PDA via the measurement of its decomposition process.

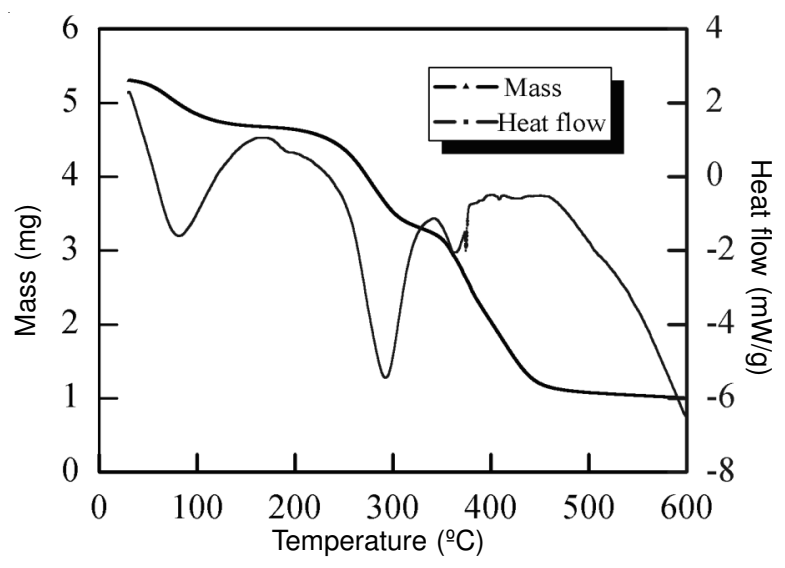

Fig. 6. TGA-DSC of thermaography of PDA 
Fig. 6 shows that DSC curve shows two endothermic peaks, the first endothermic peak between $50-100^{\circ} \mathrm{C}$, mainly the results of the high polymer absorbed water evaporates. Second endothermic peak in the $250-300{ }^{\circ} \mathrm{C}$, mainly the result of oxidation of organic matter decomposition.

The PDA lost weight in three stages. The first stage I was from $50-100^{\circ} \mathrm{C}$ mainly due to the product lost the crystal water. The second stage II was from $250-300^{\circ} \mathrm{C}$. That may be because the methyl group on the imine amide reaction and quaternary ammonium take off, take off hydrogen chloride. The third stage III was from $350-450^{\circ} \mathrm{C}$, the main chain was broken and begins to decompose. Indicating that the product is stable at room temperature.

Application of PDA for removing DBP: PDA product synthesized under the optimum conditions was used in the flocculation of the simulated water. The optimum conditions for the flocculation process investigated in this study were dosage of flocculant, $\mathrm{pH}$ value and intrinsic viscosity.

Effect of the dosage on DBP removal rate: In this experiment, the effect of PDA dosage on the DBP removal efficiency was investigated with an initial DBP concentration of 0.5 $\mathrm{mg} / \mathrm{L}$ and the dosage range from 5-9 mg/L. Not adjust the $\mathrm{pH}$ value.

Fig. 7 shows that the removal efficiency of DBP by PDA changed from a low value of $51.3 \%$ at $5 \mathrm{mg} / \mathrm{L}$ to its maximum of $83.5 \%$ at $8 \mathrm{mg} / \mathrm{L}$. That was because the mechanism of organic pollutants DBP removal in water using PDA was charge neutralization. Increased the dosage of PDA, the positive charge increased, in the result of enhanced the ability of charge neutralization, but if exceed the optimum dosage, the positive charges carried by flocculant repeled each other, resulted in the removal rate decreased.

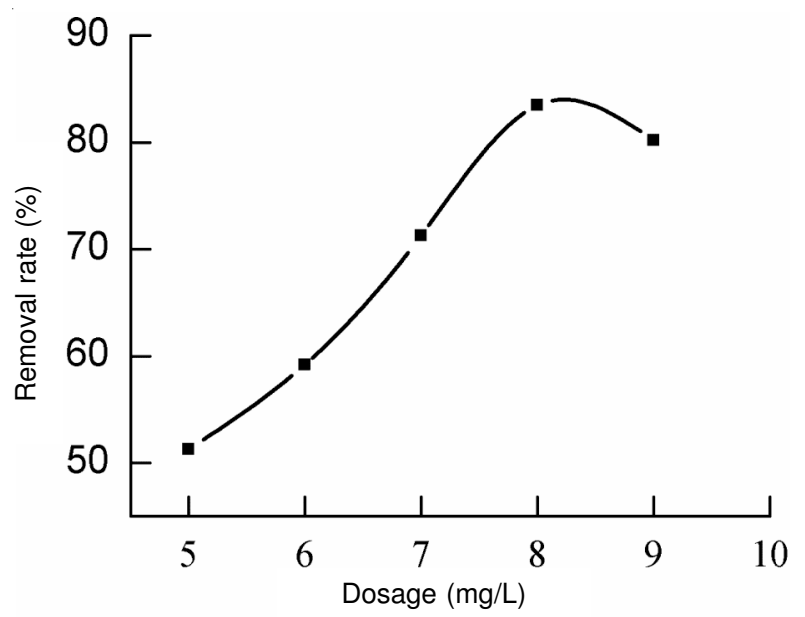

Fig. 7. Influence of dosage on the DBP removal rate

Effect of $\mathbf{p H}$ value on DBP removal efficiency: The effect of $\mathrm{pH}$ value on the DBP removal efficiency was investigated with an initial DBP concentration of $0.5 \mathrm{mg} / \mathrm{L}$.

Fig. 8 shows that the removal efficiency of DBP increases gradually with $\mathrm{pH}$ changes from $2-10$. When the $\mathrm{pH}$ value was 10.0, the maximum DBP removal efficiency of $92.4 \%$ was achieved. Therefore, the optimum $\mathrm{pH}$ value was 10 . The reason was mainly due to the wastewater $\mathrm{pH}$ value impacting on the zeta potential of the surface charge of colloidal particles

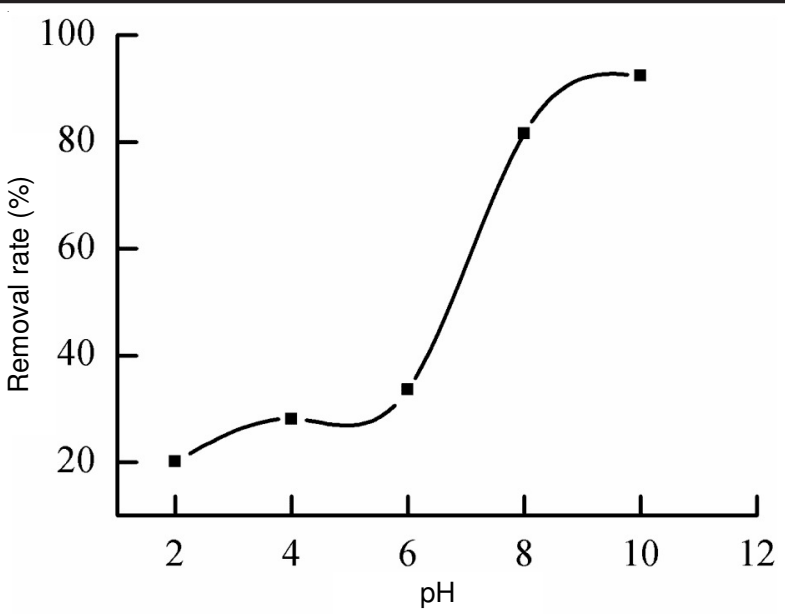

Fig. 8. Effect of $\mathrm{pH}$ value on DBP removal rate

and the hydrolysis product of flocculants. PDA used in this investigation carried a positive charge and when dissociating in water, the stronger charge neutralization and polymerization bridge chain between the flocculants and the wastewater resulted in better flocculation effects.

Effect of the intrinsic viscosity on DBP removal efficiency: The effect of the intrinsic viscosity was investigated with an initial DBP concentration of $0.5 \mathrm{mg} / \mathrm{L}$, not adjust the $\mathrm{pH}$ value.

Fig. 9 shows that the removal efficiency of DBP increased gradually with intrinsic viscosity increased. That was due to the flocculant which intrinsic viscosity was greater, the molecular of the flocculant would have the more positive charge and the longer molecular chain, leaded to the flocculant has the favorable abilities of charge neutralization and absorption-bridging.

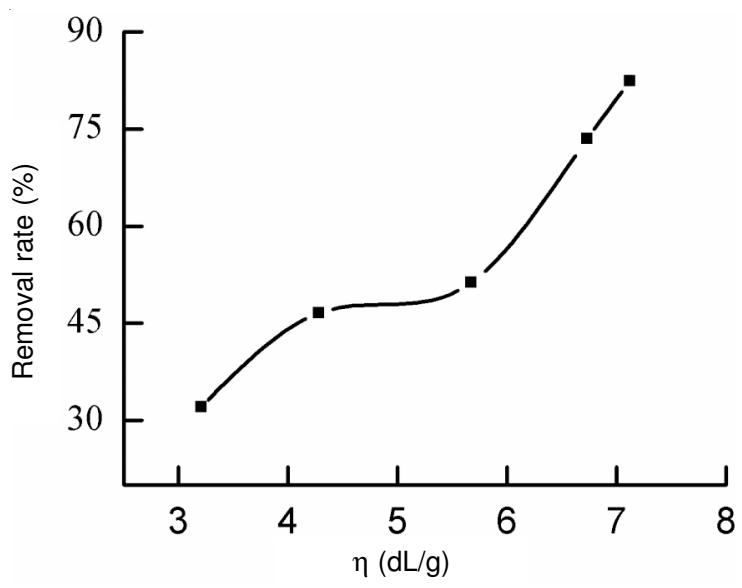

Fig. 9. Effects of intrinsic viscosity on removal of DBP

\section{Conclusion}

In this study, polydimethyl diallyl ammonium chloride acrylamide copolymer (PDA) has been formed by using industrial dimethyl diallyl ammonium chloride (DMDAAC) and industrial acrylamide (AM) initiated by $\mathrm{K}_{2} \mathrm{~S}_{2} \mathrm{O}_{8}-\mathrm{NaHSO}_{3}$, the product was detected and characterized with IR spectroscopy and thermogravimetric analysis and the DBP removal efficiency by PDA was investigated. During the experiments, the most predominant factors was adsorption of the DBP in 
simulated water, within a certain dosage range. The DBP removal efficiency increased with increasing the dosage of flocculant and the maxmium removal rate was achieved around $8.0 \mathrm{mg} / \mathrm{L}$. In the batch mode of studies, the $\mathrm{pH}$ value and effect of the intrinsic viscosity of product were also investigated. When the initial concentration of simulated water was $0.5 \mathrm{mg} / \mathrm{L}$, $\mathrm{pH}$ value of 10 , dosage of PDA was $8 \mathrm{mg} / \mathrm{L}$, the removal efficiency of DBP reached a maximum value $92.4 \%$.

\section{ACKNOWLEDGEMENTS}

The authors are grateful for the financial support provided by the National Natural Science Foundation of China (Project No.NSFC, 51174090) and the Education Department Key Project of Hunan Province (08A019).

\section{REFERENCES}

1. D.S. Wang, W. Sun, Y. Xu, H.X. Tang and J. Gregory, Colloid. Surf. A, 243, 1 (2004).

2. H.L. Zheng, X.P. Sun, Q. He, K. Liang and P. Zhang, J. Appl. Polym. Sci., 110, 2461 (2008).
3. A.T. Horvath, A.E. Horvath, T. Lindstrom and L. Wågberg, Langmuir, 24, 7857, (2008).

4. R. Losada and C. Wandrey, Macromolecules, 42, 3285, (2009).

5. T. Wintgens, M. Gallenkemper and T. Melin, Desalination, 146, 387 (2002).

6. F. Tezcan and F. Erim, Anal. Chim. Acta, 617, 196 (2008).

7. Y. Zhang, Y. Ren, H. Zhao and Y. Zhang, Anal. Chim. Acta, 584, 322 (2007).

8. W. Brostow, H.E. Hagg-Lobland, S. Pal and R.P. Singh, J. Mater. Educ. 31, 157 (2009).

9. B.L. Yuan, X.Z. Li and N. Graham, Chemosphere, 72, 197 (2008).

10. R. Losada and C. Wandrey, Macromolecules, 42, 3285 (2009).

11. R. Losada and C. Wandrey, Macromol. Rapid Commun., 29, 252 (2008).

12. M. Kobayashi, Water Res., 39, 3273 (2005).

13. Y. Suzuki and T. Maruyama, Water Res., 36, 2195 (2002).

14. Y.B. Zeng and J.B. Park, Colloid. Surf. A, 334, 147 (2009).

15. A.I. Zouboulis and P.A. Moussas, Desalination, 224, 307 (2008).

16. Y. Zheng and A.Q. Wang, J. Chem. Eng. Data, 55, 3494 (2010). 